Departamento de Ciência e Tecnologia, Secretaria de Ciência, Tecnologia e Insumos Estratégicos, Ministério da Saúde

Correspondência | Correspondence:

Decit - Departamento de Ciência e Tecnologia do Ministério da Saúde

Esplanada dos Ministérios

Bloco G sala 845

70058-900 Brasília, DF, Brasil

Texto de difusão técnico-científica do

Ministério de Saúde.

\section{Consolidação da área de avaliação de tecnologias em saúde no Brasil}

\section{Consolidation of health technology assessment in Brazil}

No âmbito do Ministério da Saúde, a estruturação da área de avaliação de tecnologias em saúde (ATS) foi iniciada com a criação do Conselho de Ciência, Tecnologia e Inovação em Saúde (CCTI), em 2003. O Conselho é responsável pela condução de diretrizes e pela promoção da avaliação tecnológica para incorporação de novos produtos e processos pelos gestores, prestadores e profissionais dos serviços no Sistema Único de Saúde (SUS).

O CCTI criou o Grupo Permanente de Trabalho em Avaliação de Tecnologias em Saúde (GT/ATS), coordenado pelo Departamento de Ciência e Tecnologia (Decit), para desenvolver estudos de ATS. ${ }^{a} \mathrm{O}$ grupo é constituído por representantes das Secretarias: de Atenção à Saúde; de Ciência, Tecnologia e Insumos Estratégicos; de Gestão do Trabalho e Educação em Saúde; de Vigilância em Saúde; e Executiva; do Conselho Nacional de Secretários de Saúde (Conass), do Conselho Nacional de Secretários Municipais de Saúde (Conasems); da Agência Nacional de Vigilância Sanitária (Anvisa); e da Agência Nacional de Saúde Suplementar (ANS).

Dentro de sua estrutura, o Decit criou, em 2005, uma área voltada para a ATS, para consolidar o trabalho desenvolvido pelo GT/ATS, com os seguintes propósitos: promover estudos de ATS para subsidiar a tomada de decisão no SUS, monitorar a utilização de tecnologias já incorporadas ao SUS, capacitar os gestores e profissionais de saúde e disseminar resultados da ATS para os gestores.

Como desdobramento dessas atividades de estruturação da ATS no Brasil, em 2009, foram ampliadas as atividades da Rede Brasileira de Avaliação de Tecnologias em Saúde (Rebrats), foi aprovada a Política Nacional de Gestão de Tecnologias em Saúde (PNGTS) e con- quistado o direito de sediar o Encontro Anual do Health Technology Assessment International (HTAi) do ano de 2011, no Rio de Janeiro.

\section{Fortalecimento da Rebrats}

Lançada em 2008, a Rebrats tem por princípio o estabelecimento de elos entre pesquisa, política, gestão e pesquisa. Coordenada pelo Decit, a rede reúne instituições gestoras do SUS, instituições de ensino e pesquisa, unidades de saúde, hospitais, sociedades profissionais e de usuários.

Entre seus objetivos principais, a Rebrats busca: produzir e disseminar estudos e pesquisas prioritárias no campo de ATS, padronizar metodologias, monitorar o horizonte tecnológico, validar a qualidade dos estudos e promover o uso de evidência científica para o processo de tomada de decisão em saúde.

Alguns resultados das atividades desenvolvidas pelos grupos de trabalho da rede foram: a implantação de Núcleos de Avaliação de Tecnologias em Saúde (NATS) em hospitais públicos de ensino, a convocatória pública para desenvolvimento de estudos de ATS e o lançamento do sistema de informação da Rebrats.

Os hospitais são os grandes utilizadores de tecnologias no sistema de saúde pois os gestores hospitalares necessitam de informações coerentes sobre benefícios, riscos e custos das tecnologias e seu impacto. Nesse contexto, a criação dos NATS possibilitará a promoção e a disseminação da cultura de ATS nessas instituições, a capacitação de seus profissionais, a utilização de seus instrumentos para avaliação de intervenções de diagnóstico, prevenção e tratamento e a criação de diretrizes terapêuticas baseadas em evidências visando ao uso racional de tecnologias e à segurança do paciente.

a No âmbito do GT/ATS e da área de ATS do Ministério da Saúde, foram realizados alguns estudos, disponíveis em: http://portal.saude.gov.br/ portal/saude/profissional/visualizar_texto.cfm?idtxt=25514 
Para viabilizar a criação dos NATS, o Decit e a Anvisa estabeleceram uma parceria e realizaram chamada pública em junho de 2009. De 54 propostas recebidas, 24 hospitais foram selecionados, de todas as regiões do Brasil, e destinados recursos para o financiamento de atividades para o primeiro semestre de 2010. As atribuições dos NATS são: promover capacidade técnica para a inserção de instituições na Rebrats; desenvolver ações para a capacitação permanente de profissionais; incentivar e produzir pesquisa voltada ao uso da evidência científica na tomada de decisão; coordenar a revisão de diretrizes clínicas dos hospitais, em consonância com as necessidades do sistema púbico de saúde; sensibilizar e incentivar os profissionais dos hospitais à introdução da cultura de ATS; e fomentar a articulação entre ensino e serviço na área de ATS.

Edital lançado pelo Ministério da Saúde em 2009, fruto da parceria interinstitucional com o Ministério da Ciência e Tecnologia (MCT) por meio do Conselho Nacional de Desenvolvimento Científico e Tecnológico (CNPq), permitirá o desenvolvimento de pesquisas em ATS no Brasil. Esse edital, a quarta convocatória pública para ATS, disponibilizou recursos ao atendimento de 33 temas de pesquisa em tópicos como doenças infecciosas e neoplasias, para diversos desenhos metodológicos, entre eles avaliações econômicas em saúde e revisões sistemáticas. Destaca-se que, nesse último edital, metade dos recursos investidos foi do próprio MCT, numa iniciativa pioneira para a área de ATS no País.

A idealização do Sistema de Informação da Rebrats surgiu da necessidade de incrementar a interlocução entre os membros da rede, bem como servir de veículo de disseminação do conhecimento consolidado para a sociedade. O Sistema, que recebe estudos de pesquisadores vinculados a instituições membros da Rebrats, está disponível na Internet, desde novembro de 2009. ${ }^{a}$ Os estudos cadastrados são comentados por especialistas da área por meio do "Parecer Rebrats", que expressa, de forma clara e sucinta, a relevância do estudo para o SUS e favorece a conexão entre pesquisa, política e gestão para subsidiar o processo decisório, oportunamente, nas fases de incorporação, monitoramento e abandono de tecnologias nos contextos da atenção à saúde. $\mathrm{O}$ acesso às informações do sistema é livre.

O fortalecimento da Rebrats como uma estratégia para viabilizar a elaboração e a disseminação de estudos prioritários de ATS para o sistema de saúde faz parte do contexto de aprimoramento da capacidade regulatória do Estado, previsto na Política Nacional de Ciência, Tecnologia e Inovação em Saúde. ${ }^{\mathrm{b}}$ A rede, com seus centros colaboradores e instituições de ensino e pesquisa, também está inserida na Política Nacional de Gestão de Tecnologias em Saúde (PNGTS), tendo apoiado o Decit na geração e na síntese de evidências científicas no campo de ATS.

\section{Aprovação da Política Nacional de Gestão de Tecnologias em Saúde}

Publicada em novembro de 2009, a Portaria no 2690/ GM, ${ }^{c}$ que instituiu a PNGTS, é fruto de uma construção coletiva, e suas bases foram lançadas pela publicação da Portaria $\mathrm{n}^{\circ}$ 2.510/GM, ${ }^{\mathrm{d}}$ de dezembro de 2005, que instituiu a Comissão para Elaboração de Proposta de Política de Gestão de Tecnologias no âmbito do SUS.

A PNGTS foi submetida à sociedade brasileira para consulta pública em outubro de 2006 e amplamente discutida na Comissão Intersetorial de Ciência e Tecnologia (CICT) do Conselho Nacional de Saúde (CNS), recebendo contribuições relevantes do Conass e Conasems.

Este esforço de quatro anos resultou na pactuação com as três esferas de Governo na Comissão Intergestores Tripartite e, posteriormente, com a sociedade, em aprovação unânime dos conselheiros do CNS.

A PNGTS define gestão de tecnologias em saúde como o conjunto de atividades gestoras relacionadas com os processos de avaliação, incorporação, difusão, gerenciamento de utilização e retirada de tecnologias do sistema de saúde. Seu objetivo geral é maximizar os benefícios de saúde a serem obtidos com os recursos disponíveis, assegurando o acesso da população a tecnologias efetivas e seguras, em condições de eqüidade.

As diretrizes dessa Política são: utilização de evidências científicas para subsidiar a gestão por meio da avaliação de tecnologias em saúde; aprimoramento do processo de incorporação de tecnologias; racionalização da utilização de tecnologias; apoio ao fortalecimento do ensino e pesquisa em gestão de tecnologias em saúde; sistematização e disseminação de informações; fortalecimento das estruturas governamentais; e articulação político-institucional e intersetorial.

A implantação plena da Política é obrigatória para o Ministério da Saúde e será coordenada pelo Decit. Para a Anvisa, a ANS, os municípios, os estados e o Distrito Federal, as suas diretrizes têm caráter recomendatório.

\footnotetext{
a Rede Brasileira de Avaliação de Tecnologias em Saúde. Disponível em: www.saude.gov.br/sisrebrats.

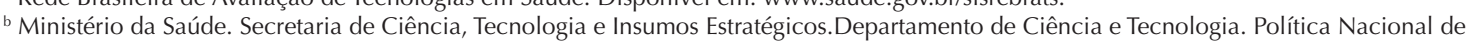
Ciência, Tecnologia e Inovação em Saúde. 2.ed. Brasília; 2006[citado 2010 fev 10]. (Série B. Textos Básicos em Saúde). Disponível em: http:// bvsms.saude.gov.br/bvs/ct/pdf/politica_portugues.pdf

${ }^{c}$ Ministério da Saúde. Portaria n 2690/GM de 05 de novembro de 2004. Institui, no âmbito do Sistema Único de Saúde (SUS), a Política Nacional de Gestão de Tecnologias em Saúde. Diario Oficial Uniao. 6 nov 2009;Seção1:61.

d Ministério da Saúde. Portaria n 2.510/GM, de 19 dezembro de 2005. Institui Comissão para Elaboração da Política de Gestão Tecnológica no âmbito do Sistema Único de Saúde - CPGT. Diario Oficial Uniao. 20 dez 2005;Seção1:77.
} 
Os entes federados poderão, ainda, complementar a PNGTS para atender às necessidades e peculiaridades locais e regionais.

\section{Encontro do Health Technology Assessment International no Brasil}

O HTAi é um evento científico que objetiva apoiar o desenvolvimento, o uso e a disseminação da ATS no mundo, como forma de promover a introdução de inovações efetivas e a efetividade do uso de recursos na atenção à saúde. A cidade do Rio de Janeiro foi escolhida para sediar o $8^{\circ}$ Encontro Anual do HTAi, em junho de 2011.
Esta será a primeira vez que o maior evento na área de ATS ocorrerá na América Latina. Com o tema "ATS e sustentabilidade dos sistemas de saúde nos próximos 10 anos", o evento será organizado por: Decit, Anvisa, ANS, Centro Cochrane do Brasil, Fundação de Amparo à Pesquisa do Estado do Rio de Janeiro, Organização Pan-Americana da Saúde e membros da Rebrats.

Desde 2004, o HTAi promove encontros anuais, os quais proporcionam importante fórum de debate para pesquisadores, profissionais de saúde e gestores compartilharem experiências e conhecimento sobre as melhores práticas em ATS para o processo de tomada de decisão em saúde. Em 2010, o encontro ocorrerá em Dublin, na Irlanda. 